\title{
Quadrilateral space syndrome: a case study and review of the literature
}

\author{
W T Hoskins, H P Pollard, A J McDonald
}

Br J Sports Med 2005;39:e9 (http://www.bjsportmed.com/cgi/content/full/39/2/e9). doi: 10.1136/bjsm.2004.013367

Quadrilateral space syndrome is an uncommon injury. The true prevalence is unknown because of a lack of literature and possible misdiagnosis. Prevalence may increase as knowledge of the syndrome increases. The case is presented of a recreational triathlete who had a spontaneous onset of quadrilateral space syndrome. The diagnosis was made by physical examination and confirmed with magnetic resonance imaging. A conservative, yet aggressive rehabilitation programme resulted in functional improvement within six weeks. Results have been maintained for eight weeks.

$\mathrm{T}$ he quadrilateral space (QS) is the anatomical compartment formed by the teres major inferiorly, the long head of triceps medially, the teres minor posteriorly, the subscapularis anteriorly, and the surgical neck of humerus laterally. ${ }^{1}$ Quadrilateral space syndrome (QSS) is a neurovascular compression syndrome of the posterior humeral circumflex artery (PHCA) and/or the axillary nerve or one of its major branches in the QS. ${ }^{2}{ }^{3}$ Table 1 presents a diagnostic summary.

\section{CASE STUDY}

A 50 year old male, recreational triathlete (right hand dominant) presented with spontaneous onset of acute left posterolateral shoulder pain, with weakness of external rotation and abduction. He had a 10 year history of episodic left shoulder symptoms. Clinical examination showed acute pain on attempted shoulder abduction, external rotation, and extension. Passive abduction and external rotation aggravated the symptoms. Mild atrophy of the deltoid and infraspinatus was noted. There was no localised swelling or masses present, and vital signs were normal. On muscle testing, weakness was graded $2 / 5$ for external rotation and $3 / 5$ for abduction. Neurological, drop arm, shoulder relocation, apprehension, impingement, and cervical and thoracic outlet testing were all negative, as was palpation of the supraspinatus and biceps tendons.

Shoulder radiographs were taken to check for osteoarthritic change, which were normal. Standard sequence magnetic resonance imaging (MRI) was performed, which indicated mild fatty involution of the teres minor without cyst formation. No change was noted within the infraspinatus or supraspinatus. There was no full thickness rotator cuff tear. There was prominent cystic change at the anterior aspect of the humeral greater tuberosity associated with minor partial thickness intrasubstance tear of the supraspinatus insertion. There was also some bursal surface irregularity distally and minor subacromial/subdeltoid bursitis. Although no abnormality could be seen within the QS, the appearance suggested QSS.

The patient was treated conservatively with ice and physiotherapy. This included transverse friction massage and active release soft tissue massage techniques to the QS, mobilisation of the humerus into internal rotation, shoulder mobilisations directed at a tight posterior capsule; the patient was also advised to start stretching of the shoulder into horizontal adduction and internal rotation. This continued for one month. After two weeks, there was moderate infraspinatus and deltoid atrophy, and the patient was using compensatory muscle actions, adopting a posture of a high left shoulder and left tilted head. The patient began performing active shoulder range of motion exercises and posterior rotator cuff strengthening, beginning with isometrics. Spinal manipulative therapy was given to the upper thoracic and cervical spine, and soft tissue massage was applied to the upper trapezius and pectoral muscles. These treatments collectively resulted in symptomatic relief of back and neck pain. After four weeks, strengthening progressed to concentrics with a theraband. After six weeks, the patient was pain free and had near total functional improvement, despite some remaining external rotation weakness (graded 4/5) and abduction weakness (graded 4/5). Long term rehabilitation for a three month period was advised, including scapular stabilisation exercises. Follow up eight weeks later showed continual improvement in strength and shoulder range of movement, and the patient had started swim training again without incident.

\section{DISCUSSION}

Entrapment of the axillary nerve occurs with QSS, but injury is most commonly associated with humeral fracture or dislocation. ${ }^{4}$ The pathophysiology of QSS remains unclear. Some believe the dominant aetiological mechanism is neurological entrapment, ${ }^{5}$ whereas others suggest that vascular occlusion ${ }^{6}$ is dominant. Compression may be static or dynamic ${ }^{4}$ and it mainly affects young active adults. ${ }^{5}$ QSS should be considered in patients with shoulder pain of unknown aetiology ${ }^{7}$ or with neck and shoulder pain and QS tenderness. ${ }^{8}$ The differential diagnosis includes thoracic outlet syndrome, referred pain from neck structures, rotator cuff syndrome, and impingement syndrome. ${ }^{9}$

QSS most commonly occurs when the neurovascular bundle is compressed by fibrotic bands within the QS as it traverses the $\mathrm{QS}^{5}{ }^{10}$ or by hypertrophy of the muscle boundaries. $^{311}$ A combination may occur. Fibrotic bands form as the result of trauma, with resultant scarring and adhesions. However, these changes have not been found in cadaver dissections. ${ }^{12}$ Cases reported in throwing athletes, ${ }^{6}{ }^{13}$ tennis players, ${ }^{10}$ and in the dominant arm of volleyball players $^{1}$ exist to support fibrosis and hypertrophy based mechanisms. Variation in axillary nerve division ${ }^{14}$ and a genetically smaller $\mathrm{QS}^{11}$ have been hypothesised to predispose

Abbreviations: $\mathrm{MRI}$, magnetic resonance imaging; $\mathrm{PCHA}$, posterior humeral circumflex artery; QS, quadrilateral space; QSS, quadrilateral space syndrome 
Table 1 Quadrilateral space syndrome physical examination diagnostic summary ${ }^{2}$

- Point tenderness to the quadrilateral space

- Poorly localised shoulder pain and/or parathesias radiating nondermatomally to the lateral shoulder and arm

- Forced abduction and external rotation aggravate the symptoms

- Deltoid and teres minor weakness with possible atrophy

- Intermittent claudication symptoms

to QSS. This may account for the limited number of reported cases.

Other reported cases of QSS include those associated with acute trauma to the shoulder ${ }^{3}$ and spontaneous occurrence without trauma. ${ }^{7}$ Pathology has resulted in QSS due to a ganglion, ${ }^{15}$ glenoid labral cyst, ${ }^{16}$ and a paralabral cyst arising from a detached inferior glenoid labral tear. ${ }^{17}$

Imaging is required to confirm diagnosis and prevent unnecessary surgery, as QSS can mimic rotator cuff or labral abnormalities. Best practice is not definitive. MRI should be favoured, as QSS has a distinctive appearance of selective teres minor fatty atrophy ${ }^{10}{ }^{17} 18$ which may not be clinically evident on examination. ${ }^{10}$ MRI also excludes pathological causes. Subclavian arteriogram or angio-MRI with the arm in abduction and external rotation ${ }^{25}{ }^{19}$ to reveal PHCA occlusion which does not occur in neutral ${ }^{7}$ have also been used. In the only controlled study, PHCA occlusion was seen in $80 \%$ of asymptomatic controls $(n=6) .{ }^{19}$ This casts doubt on the validity of arteriography, despite it being described as the cornerstone of diagnosis. ${ }^{2}$ Another study found arteriography not necessary to make the diagnosis. ${ }^{3}$ Postoperative arteriography cases have not been reported. Electromyographic and nerve conduction studies have been used, producing inconsistent results ${ }^{23}$ and are considered non-specific.

Management literature and reports have been limited. The consensus is that initial treatment should be conservative, comprising analgesics, physiotherapy, and avoidance of athletic activities. ${ }^{45}$ Physiotherapy should include cross friction and active release soft tissue massage techniques to the QS, internal mobilisation of the humerus, stretching into horizontal adduction and internal rotation, and posterior rotator cuff strengthening. ${ }^{20}$ Shoulder joint contracture should be avoided, as it may affect functional outcome despite a return of nerve function. ${ }^{21}$ Suspension or reduction of physical activity has resulted in clinical improvement. ${ }^{11}$ Healing should occur at a good rate because of the relatively short distance between the zone of injury and motor end plate. ${ }^{22}$

Chen $\mathrm{et}_{\mathrm{al}}^{8}$ suggest that surgical decompression should be started as soon as the diagnosis is made. Surgery is usually reserved for those suffering acute or chronic symptoms not responding to conservative care. ${ }^{45} \mathrm{~A}$ posterior approach, sparing the deltoid and teres minor muscles, to decompress the QS with lysis of fibrous tissue is preferential. ${ }^{4}$ Results of surgery have been positive, with one study of 18 patients having eight with complete relief, eight with improvement, and two with no improvement. ${ }^{12}$ Others have reported relief of symptoms, although functional return in some cases was minimal.

\section{CONCLUSION}

The clinician needs to be aware of the diagnostic features and differential diagnosis of QSS to ensure an accurate diagnosis. This will prevent unnecessary surgery and allow effective treatment to return the patient to full activities.

\section{Authors' affiliations}

W T Hoskins, H P Pollard, Department of Health and Chiropractic, Macquarie University, NSW 2109, Australia

A J McDonald, South Sydney Sports Medicine, Kensington, NSW 2033, Australia

Competing interests: none declared

Correspondence to: Mr Hoskins, Department of Health and Chiropractic, Macquarie University, NSW 2109, Australia; waynehoskins@optushome.com.au

Accepted 20 July 2004

\section{REFERENCES}

1 Liveson J. Nerve lesions associated with shoulder dislocation: an electrodiagnostic study of 11 cases. I Neurol Neurosurg Psychiatry 1984;47:742-4.

2 Chautems RC, Glauser T, Waeber-Fey MC, et al. Quadrilateral space syndrome: case report and review of the literature. Ann Vasc Surg 2000;14:673-6.

3 Francel TJ, Dellon AL, Campbell JN. Quadrilateral space syndrome: diagnosis and operative decompression technique. Plast Reconstr Surg 1991:87:911-16.

4 Perlmutter GS. Axillary nerve injury. Clin Orthop 1999;(368):28-36.

5 Lester B, Jeong GK, Weiland AJ, et al. Quadrilateral space syndrome: diagnosis, pathology, and treatment. Am J Orthop 1999;28:718-22, 725.

6 Dugas JR, Weiland AJ. Vascular pathology in the throwing athlete. Hand Clin 2000;16:477-85.

7 Okino S, Miyaji H, Matoba M. The quadrilateral space syndrome. Neuroradiology 1995;37:311-12.

8 Chen D, Cai P, Lao G, et al. Quadrilateral space syndrome. Chin Med J (Engl) 1995;108:109-12.

9 Zlatkin MB, Reicher MA, Kellerhouse LE, et al. The painful shoulder: MR imaging of the glenohumeral joint. J Comput Assist Tomogr 1988; 12:995-1001

10 Linker CS, Helms CA, Fritz RC. Quadrilateral space syndrome: findings at MR imaging. Radiology 1993;188:675-6.

11 Paladini D, Dellantonio R, Cinti A, et al. Axillary neuropathy in volleyball players: report of two cases and literature review. I Neurol Neurosurg Psychiatry 1996;60:345-7.

12 Cahill BR, Palmer RE. Quadrilateral space syndrome. J Hand Surg [Am] 1983:8:65-9.

13 Baker CL Jr, Liu SH. Neurovascular injuries to the shoulder. J Orthop Sports Phys Ther 1993; 18:360-4.

14 Duparc F, Bocquet G, Simonet J, et al. Anatomical basis of the variable aspects of injuries of the axillary nerve (excluding the terminal branches in the deltoid muscle). Surg Radiol Anat 1997;19:127-32.

15 Ishima $T$, Usui $M$, Satoh E, et al. Quadrilateral space syndrome caused by a ganglion. J Shoulder Elbow Surg 1998;7:80-2.

16 Robinson P, White $L M$, Lax $M$, et al. Quadrilateral space syndrome caused by glenoid labral cyst. AJR Am J Roentgenol 2000;175:1103-5.

17 Sanders TG, Tirman PF. Paralabral cyst: an unusual cause of quadrilateral space syndrome. Arthroscopy 1999;15:632-7.

18 Helms CA. The impact of MR imaging in sports medicine. Radiology 2002;224:631-5

19 Mochizuki T, Isoda H, Masui T, et al. Occlusion of the posterior humeral circumflex artery: detection with MR angiography in healthy volunteers and in a patient with quadrilateral space syndrome. AJR Am J Roentgenol 1994; 163:625-7

20 Hammer, WI. Functional soft tissue examination and treatment by manual methods, 2nd ed. Gaithersburg, MD: Aspen Publishers Inc, 1999:119.

21 Perlmutter GS, Apruzzese W. Axillary nerve injuries in contact sports: recommendations for treatment and rehabilitation. Sports Med 1998;26:351-61.

22 Steinmann SP, Moran EA. Axillary nerve injury: diagnosis and treatment. J Am Acad Orthop Surg 2001;9:328-35. 